\title{
Influence of Quantum Well Barrier Height on the Amplitude-Phase Coupling and the Spectral Linewidth in Quantum Well Lasers
}

\author{
B. Zhao, T. R. Chen, J. Iannelli, Y. H. Zhuang, Y. Yamada, and A. Yariv \\ T. J. Watson Sr. Laboratories of Applied Physics, 128-95 \\ California Institute of Technology, Pasadena, CA 91125, USA
}

Recently, it has been found both theoretically and experimentally that the differential gain in quantum well lasers is significantly affected by the unavoidable thermal population of injected carriers in the upper subbands of the quantum well structure, especially in the states of the separate confinement heterostructure region (state filling effect). The differential gain depends on the rate of increase of the quasi Fermi energies with increasing injected carrier density. The presence of upper subbands with large density of states tends to clamp the Fermi energies thus leading to a low differential gain. The state filling effect also significantly affects the amplitude-phase coupling and the spectral linewidth in quantum well lasers as a direct consequence of its influence on the differential gain.

As schematically shown in Fig.1, two GaAs/AlGaAs graded index separate confinement heterostructure (GRINSCH) single quantum well laser structures were grown by molecular beam epitaxy (MBE). The profiles and dimensions of the two GRINSCH structures are identical. The only difference between them is the different barrier height for the quantum well, which results in different state filling effects in these two structures. The calculated amplitude-phase coupling factor (or linewidth enhancement factor) $\alpha$ in these two structures are shown in Fig. 2 as a function of the modal gain. It is shown that the difference in $\alpha$ parameter can be as much as a factor of two. After the MBE growth, buried heterostructure (BH) lasers of the two different GRINSCH structures were fabricated using a liquid phase epitaxy regrowth technique combined with other conventional process techniques. The spectral linewidth of these BH lasers were measured with a measurement setup including scanning Fabry-Perot interferometer. Fig. 3 shows the linewidth as a function of inverse optical output power for uncoated BH lasers of structure A and B. The cavity lengths of these two lasers are both $300 \mu \mathrm{m}$ and the active stripe widths are about $4 \mu \mathrm{m}$. The threshold current are $12 \mathrm{~mA}$ (A) and $16 \mathrm{~mA}(\mathrm{~B})$, respectively. The high speed modulation measurement of these lasers revealed that the differential gain in the laser of structure $A$ is about three times as large as that in the laser of structure B. The spontaneous emission factor are estimated to be nearly identical in these two lasers. Thus the different spectral characteristics in these two lasers are mainly due to the different state-filling caused different amplitude-phase coupling.

In conclusion, we have found that the state filling effect also has a significant impact on the amplitude-phase coupling and the spectral characteristics in quantum well lasers due to its influence on the differential gain. 


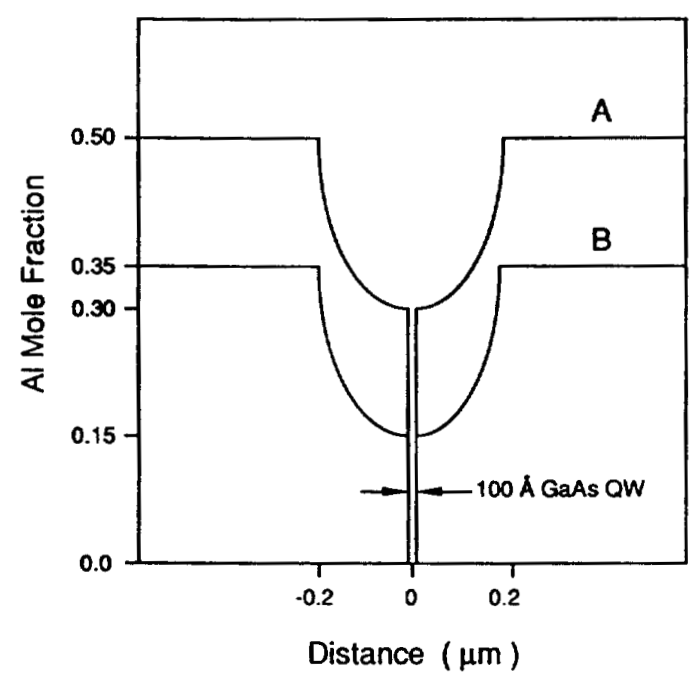

Fig.1. The schematic structure of the GaAs/AlGaAs graded index separate confinement heterostructure single quantum well lasers used in study.

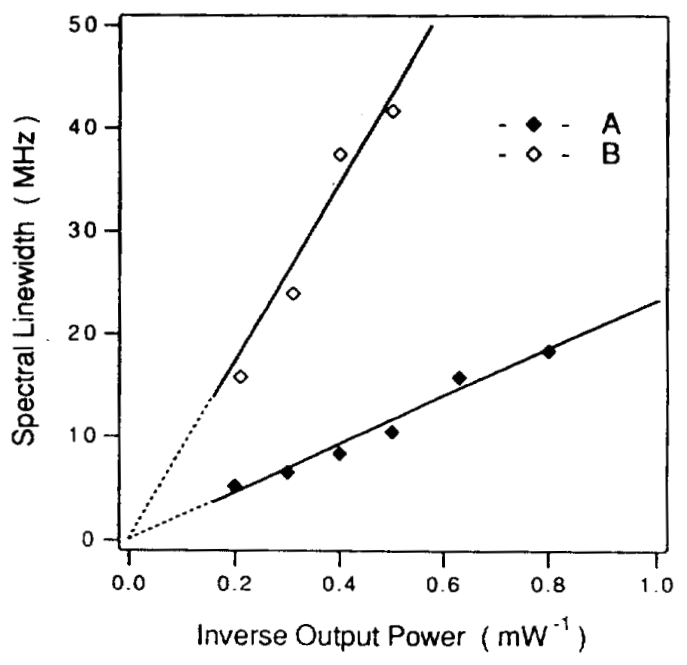

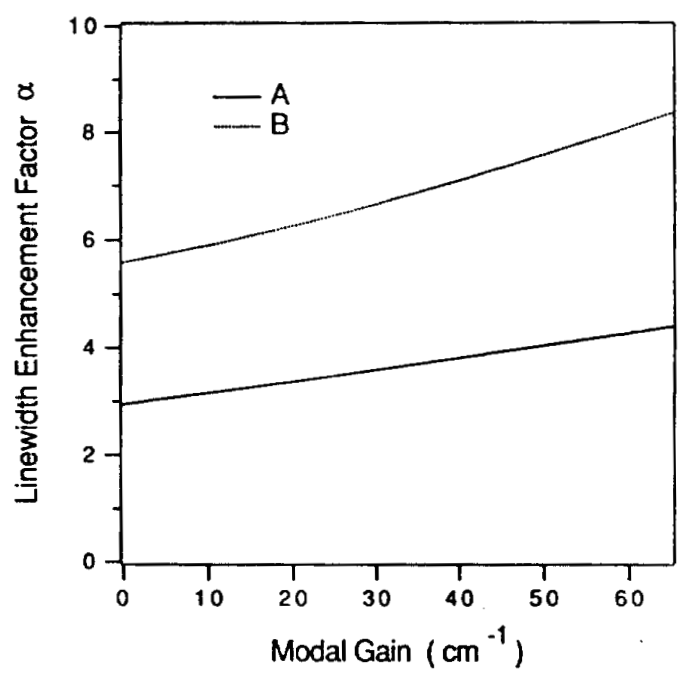

Fig.2. The calculated linewidth enhancement factor $\alpha$ as a function of the modal gain in the different structures.
Fig.3. The measured spectral linewidth $v s$. inverse optical output power for lasers of different quantum well barrier height. The cavity lengths are $300 \mu \mathrm{m}$. 